LAWRENCE LIVERMORE N A T IO N A L LABORATORY

\section{Loops in Reeb Graphs of 2-Manifolds}

Kree Cole-McLaughlin, Herbert Edelsbrunner, John Harer, Vijay Natarajan, Valerio Pascucci

December 20, 2004

Discrete and Computational Geometry 
This document was prepared as an account of work sponsored by an agency of the United States Government. Neither the United States Government nor the University of California nor any of their employees, makes any warranty, express or implied, or assumes any legal liability or responsibility for the accuracy, completeness, or usefulness of any information, apparatus, product, or process disclosed, or represents that its use would not infringe privately owned rights. Reference herein to any specific commercial product, process, or service by trade name, trademark, manufacturer, or otherwise, does not necessarily constitute or imply its endorsement, recommendation, or favoring by the United States Government or the University of California. The views and opinions of authors expressed herein do not necessarily state or reflect those of the United States Government or the University of California, and shall not be used for advertising or product endorsement purposes. 


\title{
Loops in Reeb Graphs of 2-Manifolds *
}

\author{
Kree Cole-McLaughlin ${ }^{\dagger}$, Herbert Edelsbrunner ${ }^{\ddagger}$, John Harer $^{\S}$, Vijay Natarajan ${ }^{\llbracket}$ and Valerio Pascucci ${ }^{\dagger}$
}

\begin{abstract}
Given a Morse function $f$ over a 2-manifold with or without boundary, the Reeb graph is obtained by contracting the connected components of the level sets to points. We prove tight upper and lower bounds on the number of loops in the Reeb graph that depend on the genus, the number of boundary components, and whether or not the 2-manifold is orientable. We also give an algorithm that constructs the Reeb graph in time $\mathrm{O}(n \log n)$, where $n$ is the number of edges in the triangulation used to represent the 2-manifold and the Morse function.
\end{abstract}

Keywords. Computational topology, 2-manifolds, Morse functions, level sets, Reeb graphs, loops, algorithms.

\section{Introduction}

We study Reeb graphs of Morse functions over 2-manifolds [13]. After motivating this topic and introducing the necessary definitions, we will state the results obtained in this paper.

Motivation. We are interested in the topology of smooth functions as a means to analyze and visualize intrinsic properties of geometric models and scientific data. Specifically, we study Reeb graphs, which express the connectivity of level sets. These graphs have been used in the past to construct data structures and user-interfaces for modeling and

\footnotetext{
* Research of the second and forth authors is partially supported by NSF under grants EIA-99-72879, and CCR-00-86013. Research of the third author is partially supported by NSF under grant DMS-01-07621. This work was performed under the auspices of the U. S. Department of Energy by University of California Lawrence Livermore National Laboratory under contract No. W-7405-Eng-48.

$\dagger$ Center for Applied Scientific Computing, Lawrence Livermore National Laboratory, Livermore, California.

$\ddagger$ Departments of Computer Science and Mathematics, Duke University, Durham, and Raindrop Geomagic, Research Triangle Park, North Carolina.

$\S$ Department of Mathematics and Computer Science, Duke University, Durham, North Carolina.

『 Department of Computer Science, Duke University, Durham, North Carolina.
}

visualization applications. In computer-aided geometric design, Reeb graphs have been used to describe surface embeddings up to isotopy [18]. Applications of this idea include the evolution of teeth contact interfaces in the chewing process [17]. Multi-resolution versions of the Reeb graph have lead to data-base search methods for topologically similar geometric models [8]. In the interactive exploration of scientific data, Reeb graphs are used to efficiently compute level sets [9]. Reeb graphs can also function as a user-interface tool aiding the selection of meaningful level sets [2]. A more extensive discussion of Reeb graphs and their variations in geometric modeling and visualization applications can be found in [6].

The first algorithm for constructing the Reeb graph of a smooth function over a 2-manifold is due to [16]. Given a triangulation representing the function over the 2-manifold, this algorithm may take time $\mathrm{O}\left(n^{2}\right)$, where $n$ is the number of edges in the triangulation. An improvement of the running time at the cost of accuracy has been suggested in [8]. An $\mathrm{O}(n \log n)$ time algorithm for loop-free Reeb graphs over manifolds of constant dimension has been described in [3]. For the case of 3-manifolds, this algorithm has been extended to include information about the genus of the level surfaces in [12]. In this paper, we focus on loops in Reeb graphs and study when they occur and how they can be constructed.

Definitions. Let $\mathbf{M}$ be a manifold with or without boundary. We are interested in smooth maps $f: \mathbf{M} \rightarrow \mathbb{R}$, and for simplicity in generic such maps known as Morse functions, which are defined by the following conditions:

I. all critical points of $f$ are non-degenerate and lie in the interior of $\mathbf{M}$;

II. all critical points of $f$ restricted to the boundary of $\mathbf{M}$ are non-degenerate; and

III. $f(x) \neq f(y)$ for all critical points $x \neq y$ of $f$ and its restriction to the boundary.

Compare this with the definition of a Morse function of a stratified space in the book by Goresky and MacPhearson [7, 
Section I.4]. A level set is the preimage of a constant value, $f^{-1}(t)$. The Reeb graph of $f$ is obtained by contracting the connected components of the level sets to points. An example for a 2-manifold without boundary is shown in Figure 1. Branching in the Reeb graph occurs only at nodes that cor-
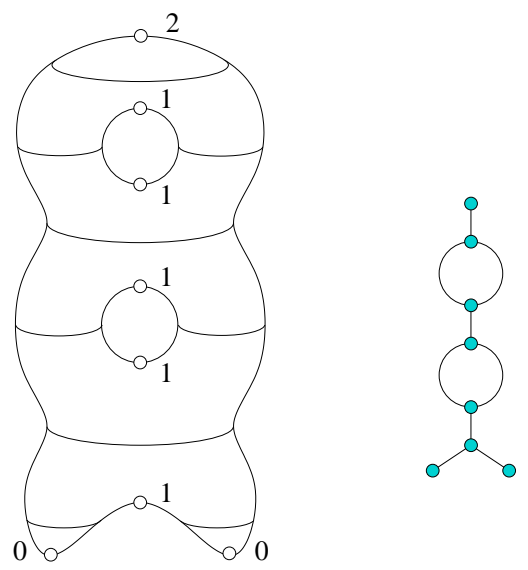

Figure 1: To the left: a double torus with $f$ equal to the height or distance from a horizontal base plane. We have $k_{0}=2, k_{1}=$ $5, k_{2}=1$ and therefore $\chi=-2$. To the right: the corresponding Reeb graph.

respond to level sets passing through critical points of $f$. We write $k_{i}$ for the number of critical points of index $i$ of $f$ and $\chi=\sum_{i}(-1)^{i} k_{i}$ for the Euler characteristic of $\mathbf{M}$.

Results. Denote the number of loops in the Reeb graph $R$ of $f$ by $\#$ loops $=\# \operatorname{loops}_{f}$. This number is equal to the first Betti number, $\beta_{1}(R)$, which is the rank of the first homology group, $\mathrm{H}_{1}(R)$. We have a continuous surjection from $\mathbf{M}$ to $R$ in which the loops in $\mathbf{M}$ that map to different loops in $R$ are neither contractible nor equivalent. It follows that the first Betti number of $\mathbf{M}$ bounds the one of $R$ from above:

$$
\text { \#loops }=\beta_{1}(R) \leq \beta_{1}(\mathbf{M})
$$

Depending on what $\mathbf{M}$ is, this bound may or may not be tight. We use combinatorial and topological arguments to get tight bounds for manifolds of dimension 2 .

According to the classification theorem of 2-manifolds, see eg. $[10,15]$, a connected orientable 2-manifold $\mathbf{M}$ is either diffeomorphic to the sphere or the connected sum of $g \geq 1$ tori, and a non-orientable 2-manifold $\mathbf{N}$ is diffeomorphic to the connected sum of $g \geq 1$ projective planes. Denoting the sphere, the torus, and the projective plane by $\mathbf{S}, \mathbf{T}$, and $\mathbf{P}$, we write

$$
\begin{aligned}
\mathbf{M} & =\mathbf{S}, \mathbf{T} \# \mathbf{T} \# \ldots \# \mathbf{T} \text { and } \\
\mathbf{N} & =\mathbf{P} \# \mathbf{P} \# \ldots \# \mathbf{P} .
\end{aligned}
$$

The Klein bottle is $\mathbf{K}=\mathbf{P} \# \mathbf{P}$, and each connected nonorientable 2-manifold is also diffeomorphic to the connected sum of an orientable 2-manifold with $\mathbf{P}$ or $\mathbf{K}$. The genus is the maximum number of pairwise disjoint simple closed curves along which we can cut while keeping the manifold in one piece. For both families, the genus is equal to $g$. We have $g=(2-\chi) / 2$ in the orientable case and $g=2-\chi$ in the non-orientable case. We get a 2 -manifold with boundary by removing $h \geq 1$ disks from one without boundary. Table 1 summarizes our results on the number of loops in the Reeb graph.

\begin{tabular}{|c|c||c|c|c|c|c|}
\hline manifold & $g$ & $h=0$ & $h=1$ & $h=2$ & $h=3$ & $\ldots$ \\
\hline \hline $\mathbf{S}$ & 0 & 0 & 0 & {$[0,1]$} & {$[0,2]$} & $\ldots$ \\
$\mathbf{T}$ & 1 & 1 & {$[1,2]$} & {$[1,3]$} & {$[1,4]$} & $\ldots$ \\
$\mathbf{T} \# \mathbf{T}$ & 2 & 2 & {$[2,4]$} & {$[2,5]$} & {$[2,6]$} & $\ldots$ \\
$\ldots$ & $\ldots$ & $\ldots$ & $\ldots$ & $\ldots$ & $\ldots$ & $\ldots$ \\
\hline $\mathbf{P}$ & 1 & 0 & {$[0,1]$} & {$[0,2]$} & {$[0,3]$} & $\ldots$ \\
$\mathbf{K}$ & 2 & {$[0,1]$} & {$[0,2]$} & {$[0,3]$} & {$[0,4]$} & $\ldots$ \\
$\mathbf{K \# \mathbf { P }}$ & 3 & {$[0,1]$} & {$[0,3]$} & {$[0,4]$} & {$[0,5]$} & $\ldots$ \\
$\mathbf{K} \# \mathbf{K}$ & 4 & {$[0,2]$} & {$[0,4]$} & {$[0,5]$} & {$[0,6]$} & $\ldots$ \\
$\ldots$ & $\ldots$ & $\ldots$ & $\ldots$ & $\ldots$ & $\ldots$ & $\ldots$ \\
\hline
\end{tabular}

Table 1: Upper and lower bounds on the number of loops in the Reeb graphs of Morse functions over orientable and non-orientable 2-manifolds with and without boundary.

To study the computational complexity of constructing a Reeb graph, we assume a piecewise linear representation of the 2-manifold and the Morse function. Letting $n$ be the number of edges in the triangulation, we give an algorithm that constructs the Reeb graph in time $\mathrm{O}(n \log n)$.

Outline. Sections 2 and 3 prove the bounds on the number of loops for orientable and non-orientable 2-manifolds with and without boundary. Sections 4 and 5 present the algorithm for constructing Reeb graphs for piecewise linear input data. Section 6 concludes this paper.

\section{Orientable 2-manifolds}

In this section, we prove tight upper and lower bounds on the number of loops in the Reeb graphs of Morse functions over orientable 2-manifolds with and without boundary.

Without boundary. Let $\mathbf{M}$ be a connected 2-manifold without boundary and $f: \mathbf{M} \rightarrow \mathbb{R}$ be a Morse function. A point of the Reeb graph $R$ of $f$ corresponds to a level set, and we refer to this point as a node if the level set passes through a critical point of $f$. The rest of the Reeb graph consists of arcs connecting the nodes. The degree of a node is the number of arcs that share the node. Since, by Condition III, all critical points have different function values, we have a bijection between the nodes of $R$ and the critical points of $f$. A minimum starts and a maximum ends a family of contour cycles, which implies that they correspond to degree-1 nodes. A saddle either splits a single cycle into two or it merges two cycles into one, and in either case it corresponds 
to a degree-3 node. Higher degrees occur only for functions $f$ that are not Morse.

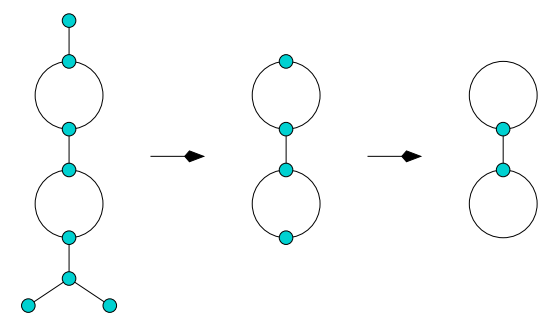

Figure 2: From left to right: the original Reeb graph of the double torus in Figure 1, the graph after collapsing, and the graph after merging arcs.

We collapse degree-1 nodes and merge arcs across degree2 nodes. Both operations preserve the homotopy type and thus the number of loops in $R$. This is illustrated in Figure 2. Let $R^{\prime}$ be the Reeb graph after collapsing and merging. Let $\ell_{3}$ be the number of degree- 3 nodes in $R^{\prime}$ and note that $\ell_{3}$ is even because $3 \ell_{3}$ is twice the number of arcs.

Case 1. $R^{\prime}$ has at least one loop. We prove - either by induction or using the Euler formula for graphs that the number of loops in $R^{\prime}$ is $\frac{\ell_{3}}{2}+1$. We have $k_{1}$ degree- 3 nodes in $R$, and for each minimum and maximum we collapse a degree- 1 node turning a degree- 3 into a degree- 2 node, which is then removed. Hence, $\ell_{3}=k_{1}-\left(k_{0}+k_{2}\right)=-\chi=2 g-2$. Since $\ell_{3}$ is a non-negative even integer, this covers the case in which $\mathbf{M}$ is the connected sum of $g \geq 1$ tori.

Case 2. $R^{\prime}$ has no loop and thus consists of a single node. Since all other possibilities are covered in Case $1, M$ is the sphere.

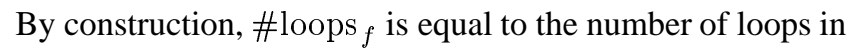
$R^{\prime}$. In both case, this number is equal to the genus:

LEMMA A. The Reeb graph of a Morse function over a connected orientable 2-manifold of genus $g$ without boundaries has $g$ loops.

A topologist's approach to proving Lemma A would be to observe that thickening the Reeb graph gives a 3-manifold $\mathbf{Q}$ whose boundary is the 2-manifold $\mathbf{M}$. Consider now the inclusion of the first homology groups, $\mathrm{H}_{1}(\mathbf{M}) \hookrightarrow \mathrm{H}_{1}(\mathbf{Q})$. By Lefschetz duality and Poincaré duality, the rank of the kernel is half the rank of the domain. We have $\operatorname{rank}_{1}(\mathbf{M})=2 g$ and therefore $\operatorname{rank} \mathrm{H}_{1}(\mathbf{Q})=2 g-g=g$, which is also the number of loops in the Reeb graph.

Note that both proofs make little use of the properties of Morse functions, implying that Lemma A holds for more general but not for arbitrary continuous functions.

With boundary. Let $\mathbf{M}$ be a connected orientable 2manifold of genus $g$ with $h \geq 1$ boundary components.
The Euler characteristic is $\chi=2-2 g-h$, which implies $\beta_{1}=2 g+h-1$. The inequality (1) implies that this is also an upper bound on the number of loops. We complement this with a lower bound to get:

LEMmA B. The Reeb graph of a Morse function over a connected orientable 2-manifold of genus $g$ with $h \geq 1$ boundary components has between $g$ and $2 g+h-1$ loops.

PROOF. Let $f$ be a Morse function over M. To prove the lower bound, we show that it is possible to close a hole without increasing the number of loops in the corresponding Reeb graph. Every boundary component is a circle and the restriction of $f$ to that circle alternates between local minima and maxima. Let $q$ be a maximum with neighboring minima $p$ and $r$. If $p=r$, we glue the two arcs by identifying points with equal function values. Otherwise, assume $f(p)<f(r)$ and let $r^{\prime}$ be the point along the arc $q p$ with $f\left(r^{\prime}\right)=f(r)$. Again we glue the arcs $q r^{\prime}$ and $q r$ by identifying points with equal function values. In both cases, the gluing starts from a single point and thus does not create any new loops (loops can only be removed). Indeed, the effect of the operation on the Reeb graph is either void or that of a zipper merging portions of two arcs starting at a common endpoint. By repeating the gluing operation we eventually remove the hole. After eliminating all $h$ holes, we are left with a function over a 2-manifold without boundary. The genus is still $g$, so by Lemma A, the Reeb graph has $g$ loops. By construction, the Reeb graph of $f$ has at least that number of loops.
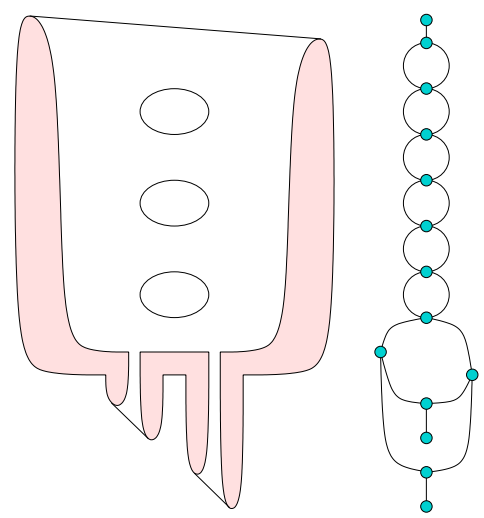

Figure 3: The outside of the blanket-like 2-manifold is white and the inside is shaded. We get two loops each for the three tunnels and one loop each for the two strips at the bottom.

The bounds in Lemma B are tight. To realize the lower bound of $g$ loops, we start with a 2-manifold without boundary and cut $h$ holes in sequence, such that no two boundary components meet a common contour cycle. To realize the upper bound of $2 g-h+1$ loops we follow the recipe illustrated in Figure 3. We start with a disk that hangs like a blanket over the back of a chair. We add $g$ tunnels in sequence and finally add $h-1$ strips at the bottom to increase the number of boundary components to $h$. 


\section{Non-orientable 2-manifolds}

In this section, we prove tight upper and lower bounds on the number of loops in the Reeb graphs of Morse functions over non-orientable 2-manifolds with and without boundary.

Without boundary. Each connected non-orientable 2manifold without boundary is the connected sum of $g$ copies of the projective plane: $\mathbf{N}=\mathbf{P} \# \mathbf{P} \# \ldots \# \mathbf{P}$. Recall that $\mathbf{K}=\mathbf{P} \# \mathbf{P}$ is the Klein bottle and that $g$ is the genus of $\mathbf{N}$. The Euler characteristic of $\mathbf{N}$ is $\chi=2-g$. We make use of a particular 2-sheeted cover of $\mathbf{N}$ obtained by doubling every point $x \in \mathbf{N}$ into a pair $x^{\prime}$ and $x^{\prime \prime}$, which we imagine as lying near $x$ and locally on opposite sides of the non-orientable manifold. Figure 4 illustrates this construction for the Klein bottle. Two points $x^{\prime}$ and $y^{\prime}$ are near if $x, y \in \mathbf{N}$ are near
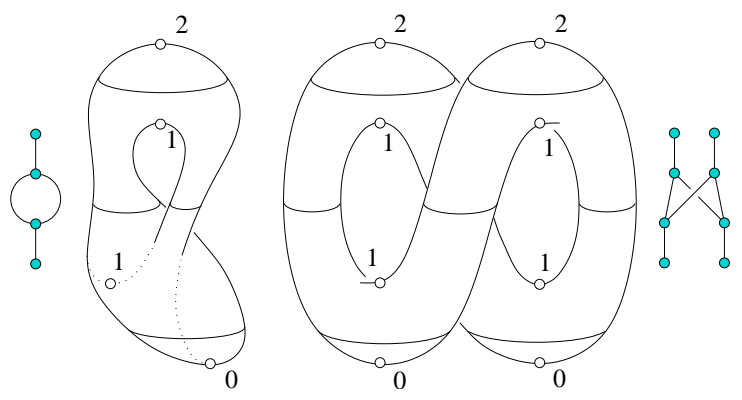

Figure 4: The Klein bottle with Reeb graph $R$ on the left and the torus obtained by doubling with its Reeb graph $R^{\prime}$ on the right.

and $x^{\prime}, y^{\prime}$ lie locally on the same side of $\mathbf{N}$. The resulting space is a connected and orientable 2-manifold $\mathrm{M}$ with $\mathrm{Eu}-$ ler characteristic $\chi_{\mathbf{M}}=2 \chi$. The genus of $\mathbf{M}$ is therefore $g_{\mathrm{M}}=\left(2-\chi_{\mathrm{M}}\right) / 2=1-\chi=g-1$. Table 2 illustrates the correspondence between the non-orientable and the orientable 2-manifolds.

\begin{tabular}{|r|r|c||c|r|r|}
\hline$\chi$ & $g$ & $\mathbf{N}$ & $\mathbf{M}$ & $g_{\mathbf{M}}$ & $\chi_{\mathbf{M}}$ \\
\hline \hline 1 & 1 & $\mathbf{P}$ & $\mathbf{S}$ & 0 & 2 \\
0 & 2 & $\mathbf{K}$ & $\mathbf{T}$ & 1 & 0 \\
-1 & 3 & $\mathbf{K \# \mathbf { P }}$ & $\mathbf{T} \# \mathbf{T}$ & 2 & -2 \\
$\ldots$ & $\ldots$ & $\ldots$ & $\ldots$ & $\ldots$ & $\ldots$ \\
\hline
\end{tabular}

Table 2: Doubling turns the non-orientable 2-manifold on the left into the orientable 2-manifold in the same row on the right.

Let $R$ be the Reeb graph of a Morse function over $\mathbf{N}$. We have seen that $R$ has degree-1 nodes corresponding to minima and maxima and degree- 3 nodes corresponding to saddles. In the non-orientable case, we also have degree2 nodes. The reason is that the level set containing a critical point of index 1 is a figure- 8 which may contain an orientation-reversing circle. A picture of the local structure for this situation is given in Figure 5. We call these critical points projective saddles since a neighborhood of the level set through such a point is the projective plane with two disks removed. The double covering $\mathbf{M} \rightarrow \mathbf{N}$ composed with the
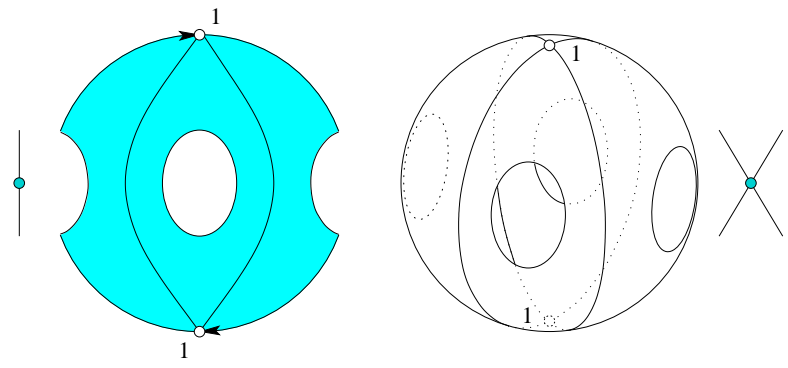

Figure 5: To the left: the projective plane with two disks removed. To the right: the 2-sheeted cover, which is a sphere with four disks removed.

Morse function $f: \mathbf{N} \rightarrow \mathbb{R}$ induces a function $f^{\prime}: \mathbf{M} \rightarrow \mathbb{R}$. Its critical points are non-degenerate, but it is not a Morse function since each critical point of $f$ corresponds to two critical points of $f^{\prime}$ at the same height. Let $R^{\prime}$ be the Reeb graph of $f^{\prime}$. Even though $f^{\prime}$ is not Morse, a small perturbation of $f^{\prime}$ is Morse and the perturbation does not alter $R^{\prime}$. By Lemma $\mathrm{A}$, the number of loops in $R^{\prime}$ is equal to the genus of M, which is $g-1$.

Contour cycles of $f$ that do not contain critical points lift to two contour cycles of $f^{\prime}$. It follows that each arc of $R$ gives rise to two arcs of $R^{\prime}$. A contour cycle of $f$ that passes through a critical point which is not a projective saddle also lifts to two disjoint copies, hence gives rise to two nodes in $R^{\prime}$. Finally, a contour cycle that contains a projective saddle lifts to a single contour cycle, giving rise to a single node connected by four arcs to other nodes in $R^{\prime}$. Now suppose that $R$ has $e$ arcs, $\ell_{2}$ degree- 2 nodes corresponding to projective saddles, and $\ell$ other nodes. Then $R^{\prime}$ has $2 e$ arcs and $2 \ell+\ell_{2}$ nodes. We have $1-\beta_{1}(R)=\ell+\ell_{2}-e$ and therefore

$$
\begin{aligned}
1-\beta_{1}\left(R^{\prime}\right) & =2 \ell+\ell_{2}-2 e \\
& =2-2 \beta_{1}(R)-\ell_{2}
\end{aligned}
$$

Since $\beta_{1}\left(R^{\prime}\right)=g-1$ we obtain $\beta_{1}(R)=\left(g-\ell_{2}\right) / 2$, which implies:

LEMma C. The Reeb graph of a Morse function over a connected non-orientable 2-manifold of genus $g$ without boundary has between 0 and $\left\lfloor\frac{g}{2}\right\rfloor$ loops.

The bounds for the number of loops are tight. Note that for the projective plane, we have $\ell_{2}=1$ and \#loops $=0$, independent of the Morse function. For general $\mathbf{N}$, we get \#loops $=0$ by connecting $g$ copies of $\mathbf{P}$ in a chain, as shown in Figure 6 but without boundary cycles. We get \#loops $=\left\lfloor\frac{g}{2}\right\rfloor$ by connecting $\left\lfloor\frac{g}{2}\right\rfloor$ copies of the Klein bottle as in Figure 4, possibly by adding another copy of $\mathbf{P}$. Numbers between the two extremes are obtained by combining the two constructions.

With boundary. Just as in the orientable case, a connected non-orientable 2-manifold $\mathbf{N}$ with boundary is characterized 
up to diffeomorphism by its genus $g$ and the number $h \geq 1$ of boundary components. Its Euler characteristic is $\chi=2-$ $g-h$. The inequality (1) implies that $\beta_{1}=1-\chi=g+h-1$ is an upper bound on the number of loops in the Reeb graph:

LEMMA D. The Reeb graph of a Morse function over a connected non-orientable 2-manifold of genus $g$ with $h \geq 1$ boundary components has between 0 and $g+h-1$ loops.
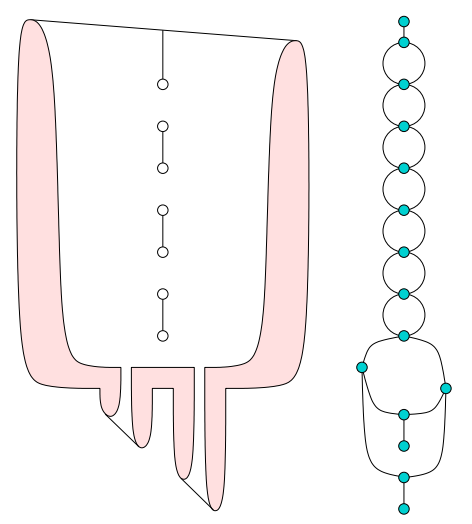

Figure 6: We get one loop each for the seven cross-caps and the two strips. The projective saddles delimiting the cross-caps are shown as small circles.

The bounds in Lemma D are tight. To see the lower bound take a Morse function over a non-orientable 2-manifold without boundary whose Reeb graph has no loop and cut $h$ holes in sequence, such that no two boundary components meet a common contour cycle. To show that the upper bound is tight, we use the construction of Figure 3 and replace tunnels by double cross-caps and, if $g$ is odd, add a single cross-cap at the top. As illustrated in Figure 6, this is done so all projective saddles have smaller function values than the blanket at the left and right shoulders.

\section{Sweep Algorithm}

In this section, we describe an algorithm that constructs the Reeb graph of a function $f: \mathbf{M} \rightarrow \mathbb{R}$. We remain on an abstract level and defer implementation details to Section 5.

Scheduling critical points. We construct the Reeb graph by maintaining the level set while sweeping $\mathbb{R}$ from $-\infty$ to $+\infty$. By definition of Morse functions, the critical points have pairwise different function values. It follows we can order them as $p_{1}, p_{2}, \ldots, p_{k}$ such that $f\left(p_{i}\right)<f\left(p_{j}\right)$ whenever $i<j$. Define $f\left(p_{0}\right)=-\infty$ and $f\left(p_{k+1}\right)=\infty$ and choose a value $t_{i}$ strictly between the function values of $p_{i}$ and $p_{i+1}$, for each $i$. Let $L_{i}=f^{-1}\left(t_{i}\right)$ be the corresponding level sets. In general, $L_{i}$ consists of finitely many closed curves (contour cycles) and curves with endpoints (contour paths). The level set remains topologically the same as long as the function value remains strictly between $f\left(p_{i}\right)$ and $f\left(p_{i+1}\right)$. The level sets in the next open interval are represented by $L_{i+1}$. The difference between $L_{i}$ and $L_{i+1}$ can be understood by studying how the level set changes when it passes through $p_{i+1}$. We distinguish between critical points of $f$ and critical points of $f$ restricted to the boundary of $\mathbf{M}$.

If $p_{i+1}$ is a minimum of $f$ then $L_{i+1}$ develops a new contour cycle. If $p_{i+1}$ is a maximum of $f$ then $L_{i+1}$ loses one contour cycle. If $p_{i+1}$ is a saddle, the evolution from $L_{i}$ to $L_{i+1}$ is more complicated because it depends on the global and not just the local connectivity of the level set. Figure 7 illustrates the various cases that can occur. On the bound-

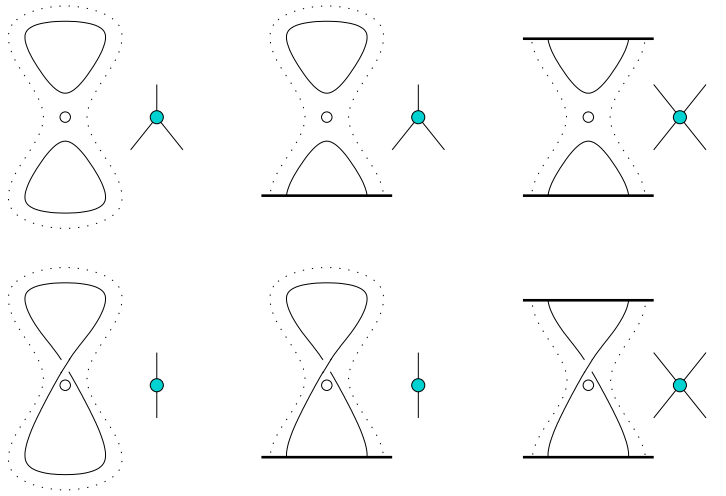

Figure 7: Evolution of the level set while passing through a (normal) saddle in the top row and a projective saddle in the bottom row. In each case, we show the corresponding new piece of the Reeb graph on the side. The solid and dotted lines show the level set before and after passing the saddle, and we get symmetric pictures (and upside down Reeb graph pieces) by switching solid with dotted lines.

ary, we have two cases per minimum and maximum. If $p_{i+1}$ is a boundary minimum, then $L_{i+1}$ either develops a new contour path or it splits a contour cycle or path open, as illustrated in Figure 8. We have the symmetric cases for boundary maxima.

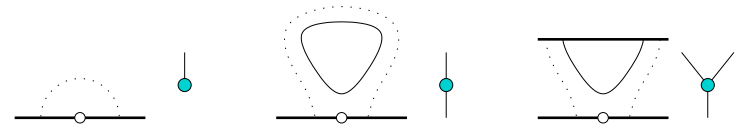

Figure 8: Evolution of the level set while passing through a boundary critical point. If that point is a minimum, the level set progresses from solid to dotted lines and the Reeb graph from bottom to top. For a boundary maximum the progress is the other way round.

Abstract data type. We store a level set as a collection of contour cycles and paths. The sweep is translated into a sequence of operations manipulating this collection. These operations create, destroy, cut, and glue cycles and paths:

MKCYCLE and MKPATH create new contour cycles and contour paths. 
RMCYCLE and RMPATH destroy old contour cycles and contour paths.

CUT splits a cycle open to a path or a path into two paths.

GLUE connects two ends of a path to form a cycle or two ends of two different paths to form one path.

To determine the effect of a cut operation, we need to be able to tell a cycle from a path. Similarly, to determine the effect of a glue operation, we need to be able to tell whether two endpoints belong to the same or to two different paths. Assuming that ability, we have an algorithm that constructs the Reeb graph of $f: \mathbf{M} \rightarrow \mathbb{R}$ by maintaining the level set during a sweep. Each interior minimum/maximum translates into a cycle creation/destruction, each interior saddle translates into two cut and two glue operations, and each boundary minimum/maximum translates into a creation/destruction or a cut/glue operation. In total, we have at most $4 k$ operations.

\section{PL Implementation}

In this section, we make the sweep algorithm of Section 4 concrete by assuming a piecewise linear input representation. It applies to the case common in practice, in which a function is only probed at a finite set of locations.

PL representation. A triangulation of a 2-manifold $M$ is a simplicial complex $K$ whose underlying space is homeomorphic to $\mathbf{M}$; see eg. $[1,15]$. Writing Vert $K$ for the collection of vertices, we use a map $\varphi:$ Vert $K \rightarrow \mathbb{R}$ and define the function $f$ over the 2-manifold $\mathbf{M}$ by linear extension over the simplices. It is clear that $f$ is not smooth and thus definitely not a Morse function. We can, however, modify $K$ and $\varphi$ such that $f$ has combinatorial properties that resemble the generic smooth properties of Morse functions. We need some definitions to explain this. The star of a vertex $u$ consists of all simplices that share $u$, the link consists of all faces of simplices in the star that do not belong to the star themselves, and the lower link is the subset of the link induced by vertices with function value less than $u$ :

$$
\begin{aligned}
\text { St } u & =\{\sigma \in K \mid u \in \sigma\}, \\
\operatorname{Lk} u & =\{\tau \in K \mid \tau \subseteq \sigma \in \text { St } u, \tau \notin \text { St } u\}, \\
\text { Lk_u } & =\{\tau \in \operatorname{Lk} u \mid v \in \tau \Longrightarrow f(v)<f(u)\} .
\end{aligned}
$$

We use the link and the lower link to classify the vertices into the various types of critical points we observe in the smooth setting. Consider first an interior vertex $u$. Its link is the cycle of edges and vertices surrounding $u$. We call $u$ regular if the lower link is a non-empty connected segment of the link; in all other cases, $u$ is critical. Specifically, $u$ is a minimum if $\mathrm{Lk}_{-} u=\emptyset, u$ is a maximum of $\mathrm{Lk}_{-} u=$ Lk $u$, and $u$ is a $k$-fold saddle if $\mathrm{Lk}_{-} u$ consists of $k+1 \geq 2$ components along the link. This is illustrated in Figure 9.

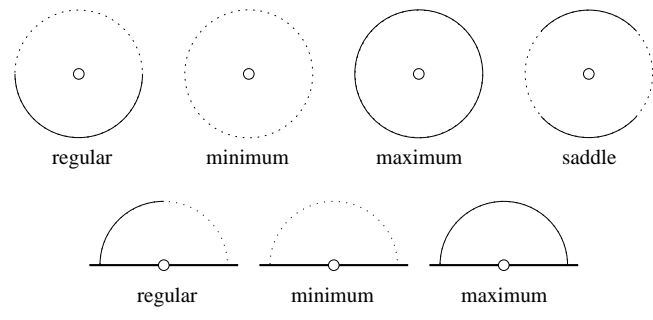

Figure 9: Regular and critical points as classified by the (solid) lower link, which is a subset of the (otherwise dotted) link.

Consider second a boundary vertex $u$. Its link is a half-circle. Along the boundary, $u$ has two neighbors $v$ and $w$, which are the endpoints of the half-circle. Assuming $f(v)<f(w)$, we call $u$ a boundary

$\left.\begin{array}{l}\text { regular point } \\ \text { minimum } \\ \text { maximum }\end{array}\right\}$ if $\left\{\begin{array}{l}f(v)<f(u)<f(w), \\ f(v)>f(u)<f(w), \\ f(v)<f(u)>f(w) .\end{array}\right.$

Figure 9 illustrates only the simple cases. Non-simple cases reduce to simple ones. For example, a $k$-fold saddle can be split into $k$ simple saddles, as described in [5]. Similarly, a boundary minimum/maximum with more complicated lower link than shown in Figure 9 can be split into simple (interior) saddles and a (simple) boundary minimum/maximum. Note that we just eliminated the rightmost two cases in Figure 8 . The corresponding smooth operation bends $\mathbf{M}$ a little near the boundary and thus creates a saddle right next to the boundary minimum/maximum. In addition to having all non-simple critical points split into simple ones, we assume $\varphi(u) \neq \varphi(v)$ for all vertices $u \neq v$ of $K$.

PL algorithm. The combinatorial structure of the piecewise linear representation of $f$ is now simple enough to allow a direct implementation of the sweep algorithm, which processes the vertices of $K$ in the sequence of increasing function values. A generic level set consists of finitely many contour cycles and contour paths. We store each cycle as a cyclic list and each path as a linear list of edges in $K$. These edges carry the vertices of the contours, and the triangles between them carry their edges. We now discuss how the collection of cyclic and linear lists changes as we pass through a vertex $u$ of $K$. If $u$ is regular, we just replace its descending edges (which all belong to a single list) by its ascending edges. In addition to the operations listed in Section 4, we therefore need the following two:

DELETE removes an edge from a cyclic or linear list.

INSERT adds an edge to a cyclic or linear list.

Similarly, if $u$ is critical, we remove its descending edges and add its ascending ones. However, now the level set changes its topology, which is reflected by structural changes in the collection of lists caused by the cut and glue operations, as described in Section 4. To identify structural changes, we use yet another operation: 
FIND determines the list that contains a given edge of $K$.

The effect of the cut and glue operations is determined by finding the lists from which the descending edges are removed and to which the ascending edges are added. By restriction to simple critical vertices, these edges belong to either one or to two lists. The corresponding local pieces appended to the Reeb graph are shown in Figure 10.

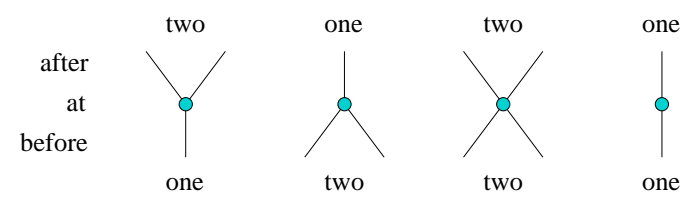

Figure 10: Adding nodes of degree 3, 3, 4, and 2 to the Reeb graph; compare with Figure 7.

Data structure and analysis. To implement all operations efficiently, we represent each list (cyclic and linear) as a balanced search tree [4]. Each insertion and deletion takes time at most logarithmic in the size of the list. Finding a given edge is implemented by walking upward to the root of the tree, which also takes at most logarithmic time. Finally, cut and glue operations are implemented by splitting and concatenating trees. Here it is important that each list is marked as either cyclic or linear, because cutting a linear list translates into splitting to generate two linear lists, while cutting a cyclic list translates into splitting and concatenating the two pieces to generate a single linear list. Similar distinctions have to be made when we glue two ends of one or two linear lists. Standard balanced search trees support split and concatenate operations again in logarithmic time. The randomized search trees developed by Seidel and Aragon [14] are particularly well suited for our purposes because they lend themselves to rather simple implementations of all necessary operations. Furthermore, experiments indicate that they outperform all alternative data structures in practice.

To give a bound on the running time of the above algorithm, we let $n$ be the number of edges in $K$. Note that $K$ contains fewer than $n$ triangles and fewer than $n$ vertices. We use $n$ insertions, $n$ deletions, and $2 n$ find operations to process all edges. Furthermore, we use at most two cut and two glue operations per vertex. Each operation takes time at most $\mathrm{O}(\log n)$, adding up to a total time of at $\operatorname{most} \mathrm{O}(n \log n)$ to construct the Reeb graph of $f$. If we use randomized search trees we get the same running time but only in the expected and not the worst case.

\section{Discussion}

In this paper, we study Reeb graphs of Morse functions over 2-manifolds with and without boundary. The number of loops is these graphs depends on the topology of the 2manifold and sometimes but not always on the Morse function. We prove tight upper and lower bounds on the number of loops and give an $\mathrm{O}(n \log n)$ time algorithm to construct a Reeb graph. It would be interesting to prove that $\mathrm{O}(n \log n)$ is indeed optimal or, alternatively, to improve the algorithm. Maybe one of the lower bounds in [11] can be extended to prove the optimality of our algorithm.

Our understanding of Reeb graphs for 3-manifolds is much less developed. It is no longer true that the number of loops for an orientable manifold without boundary is independent of the function. For example, a 3-torus can have zero or one loop in the Reeb graph. Furthermore, the existence of a Heegard decomposition shows that every 3-manifold has a Morse function whose Reeb graph is a tree. We also have no algorithm for constructing the Reeb graph of a Morse function over a 3-manifold that runs in time less than quadratic in the size of the representing triangulation.

\section{References}

[1] P. S. Alexandrov. Combinatorial Topology. Republication of three volumes published in 1956, 57, 60, Dover, Mineola, New York, 1998.

[2] C. L. Bajaj, V. Pascucci and D. R. Schikore. The contour spectrum. In "Proc. IEEE Visualization, 1997", 167175.

[3] H. Carr, J. Snoeyink and U. Axen. Computing contour trees in all dimensions. Comput. Geom. Theory Appl. 24 (2002), 75-94.

[4] T. H. Cormen, C. E. Leiserson And R. L. Rivest. Introduction to Algorithms. MIT Press, Cambridge, Massachusetts, 1994.

[5] H. Edelsbrunner, J. Harer and A. Zomorodian. Hierarchical Morse-Smale complexes for piecewise linear 2manifolds. Discrete Comput. Geom., to appear.

[6] A. T. Fomenko And T. L. Kunit, (EDS). Topological Methods for Visualization. Springer-Verlag, Tokyo, Japan, 1997.

[7] M. Goresky and R. MacPhearson. Stratified Morse Theory. Springer-Verlag, Heidelberg, Germany, 1988.

[8] M. Hilaga, Y. Shinagawa, T. Komura and T. L. KUNII. Topology matching for fully automatic similarity estimation of 3D shapes. In "Computer Graphics Proc., SIGgRAPH 2001", 203-212.

[9] M. van Kreveld, R. van Oostrum, C. L. Bajaj, V. PASCUCCI AND D. R. SCHIKORE. Contour trees and small seed sets for isosurface traversal. In "Proc. 13th Ann. Sympos. Comput. Geom., 1997”, 212-220.

[10] W. S. Massey. Algebraic Topology: An Introduction. Springer-Verlag, New York, 1967.

[11] K. Mehlhorn, S. NÄher And H. Alt. A lower bound on the complexity of the union-split-find problem. SIAM J. Comput. 17 (1988), 1093-1102. 
[12] V. Pascucci and K. Cole-McLaughlin. Efficient computation of the topology of level sets. Algorithmica, to appear.

[13] G. REEB. Sur les points singuliers d'une forme de Pfaff complèment intégrable ou d'une fonction numérique. Comptes Rendus de L'Académie ses Séances, Paris 222 (1946), 847-849.

[14] R. Seidel And C. ARAGon. Randomized search trees. Algorithmica 16 (1996), 464-497.

[15] H. SeIfERT AND W. Threlfall. Lehrbuch der Topologie. Chelsea, New York, 1934.

[16] Y. Shinagawa And T. L. KuniI. Constructing a Reeb graph automatically from cross sections. IEEE Comput. Graphics Appl. 11 (1991), 44-51.

[17] Y. Shinagawa, T. L. Kunit, H. Sato and M. Ibusuki. Modeling contact of two complex objects: with an application to characterizing dental articulations. Computers and Graphics 19 (1995), 21-28.

[18] S. Yoshinisa, T. L. Kunit and Y. L. Kergosian. Surface coding based on Morse theory. IEEE Comput. Graphics Appl. 11 (1991), 66-78. 